\title{
Computational structure in three-valued nearness relations
}

\author{
Matt Duckham and Michael Worboys \\ Department of Computer Science \\ Keele University \\ Staffordshire, ST7 8AA, UK \\ Fax: +44 1782713082 \\ Tel: +441782584270 \\ Email: matt@cs.keele.ac.uk, michael@cs.keele.ac.uk
}

\begin{abstract}
The development of cognitively plausible models of human spatial reasoning may ultimately result in computational systems that are better equipped to meet human needs. This paper explores how human subjects perceive the qualitative spatial relation nearness within an environmental space. Based on experimental data, a three-valued nearness relation is analysed in two stages. First, the results are analysed with special reference to the existence of subsets of candidate landmark places, from which nearness relations between other places may be partially inferred. Second, the desirable properties of such landmark sets are considered and some of their formal properties are presented. These properties are then considered in the light of the data furnished by the experiment. The paper concludes with a discussion of the significance of the analyses and the scope for further work in this area.
\end{abstract}

Keywords: Nearness; qualitative spatial reasoning; landmarks; data mining; similarity relation.

\section{Introduction}

The qualitative spatial relation nearness is a basic, commonly used spatial relation that is both vague and context dependent. Nearness is a vague concept in the sense that it exhibits borderline cases: it can sometimes be difficult to decide whether certain places are near or not near each other. London may be considered near Oxford and not near Edinburgh, but there exists no clearly defined boundary between Oxford and Edinburgh where places stop being near to London. Nearness is context dependent in the sense that two places can be near in one context and not near in another. In the context of capital cities of the world, London might be considered near to Paris; at the same time, in the context of cycling to work in the morning, London is certainly not near to Paris. What makes nearness particularly interesting and challenging to spatial information theorists is that despite these apparent contradictions, humans are able to 
effectively reason and communicate using vague, context dependent qualitative spatial concepts like nearness.

Research in geographic information science has been concerned primarily with quantitative rather than qualitative spatial information. By contrast, humans are generally better at qualitative rather than quantitative spatial reasoning. Many spatial concepts commonly used by humans, such as nearness, present considerable difficulties within a computational setting. For example, Fisher [4] explores some of the problems arising from the inherently vague nature of many common spatial concepts. Gahegan [8] looks at the need for a qualitative userdefined context in understanding the significance of quantitative spatial information. The practical aim of such research is ultimately the development of improved GIS interfaces that are better equipped to support human concepts and decision making processes [15], for example by developing more user-friendly spatial query languages, decision support systems, and navigational aids.

Building on experimental work reported in [25], this paper addresses the integration of experimental data and computational models concerning the qualitative spatial relation nearness. The aim of this research is to develop and explore cognitively plausible formal models of nearness as perceived by human subjects in an environmental space, the Keele University campus. Following a review of previous relevant work in $\S 2, \S 3$ presents a discussion of the experimental methods used to collect data about a group of human subjects' perception of the qualitative spatial relation nearness. The analysis of this data is presented in two stages: $\S 4$ explores the use of data mining techniques to uncover computational structure within the experimental data; $\S 5$ develops elements of this structure into a more detailed formal model of qualitative vector space. Finally, $\S 6$ presents a review of the findings and an agenda for further work.

\section{Background}

Much research into qualitative spatial reasoning can be characterised as striking a compromise between computational and cognitive perspectives. Research from a computational perspective aims to develop formal models of qualitative spatial reasoning capable of being used within computational systems. Research from a cognitive perspective aims to provide insights into how humans use and reason with qualitative spatial information, with particular reference to experiments using human subjects. Although the two perspectives are closely related they are to some extent inherently incompatible: no formal logical system will ever be able to satisfactorily capture the diversity or flexibility of human spatial reasoning. However, the attempt to develop more cognitively plausible models of human spatial reasoning depends on the closer integration of these two perspectives. This section reviews the existing research from these two perspectives, focusing in particular on the nearness relation. 


\subsection{Computational and cognitive approaches to qualitative spatial information}

In addition to inherently qualitative work on topology (eg [3]) and shape (eg [1]), work on computational aspects of qualitative distance (eg [9] and [7]) and qualitative direction (eg [14]) have appeared in the literature. Frank [6] develops a calculus usable for reasoning over both cardinal directions and qualitative distances, although the integration of distance and direction remains an unresolved research issue. A feature of many such computational approaches is that while qualitative, they often do not correspond well with the observed characteristics of human spatial cognition.

Sadalla et al. [21] observed asymmetries in the human perception of nearness, with more significant reference points or landmarks generally being understood to be near to adjacent points more frequently than vice versa. Stevens and Coupe [23] and Hirtle and Jonides [12] provide evidence of distortions in human spatial cognition resulting from the apparent hierarchical arrangement of places according to spatial and semantic criteria. Further evidence of distortions congruent with the existence of hierarchies has been observed in the form of clustering for judgements of distances between and navigational paths through sets of places $[13,11]$. Distortions such as asymmetry, vagueness, landmarks, hierarchies and clustering have proved difficult to integrate with the logical systems which form the backbone of computational approaches to qualitative spatial reasoning. Nevertheless, Tversky [24] argues that these distortions are important cognitive devices that help humans to organise spatial information. Ideally, cognitively plausible computational models of qualitative spatial information should be able to allow for such distortions.

Some research has begun to provide a basis for closer integration between the computational and the cognitive. The 'egg-yolk' calculus [2] has proved particularly useful as a formal framework for reasoning about vague or indeterminate boundaries. Hirtle [10] provides a survey of three mathematical structures (trees, ordered trees and semi-lattices) capable of representing the hierarchical nature of spatial cognition. Robinson $[18,19]$ uses an adaptive algorithm to produce a fuzzy membership function for nearness based on human subjects' responses to questions. In general, however, formal and computational work on qualitative spatial reasoning is still some way from being integrated with the observations from cognitive work. The remainder of this paper reports on work that takes a step toward providing better integration between computational and cognitive approaches to qualitative spatial reasoning.

\section{Experimentation}

The analyses explored in this paper are based upon experimental data concerning the qualitative spatial relation "near", reported in more detail in [25]. Only a brief overview of the key features and results of the experiment is given here. Twenty-two subjects were asked to complete a series of questionnaires concerning the nearness of 22 places in Keele University campus, UK. The 22 places 
were selected as being well known places on Campus, identified using a preliminary study. Half the subjects (the truth group) were asked in a questionnaire to identify which places were near to a fixed reference place, drawn from the 22 places. The other half of the subjects (the falsity group) were asked to identify which places were not near to the same reference place. So for example, 8 of the 11 people in the truth group considered Keele Hall to be near to the Library, while 2 of the 11 people in the falsity group though that Keele Hall was not near the Library. With a break of at least one day between successive questionnaires, each subject was then asked to complete further questionnaires, one for each reference place, until information about all 22 reference places had been gathered for each subject. All the subjects were Keele University staff with some years' experience of the Campus and were asked to complete questionnaires without reference to maps.

Worboys [25] identifies three different approaches to the analysis of the results of the experiment which allow for the inherently vague nature of nearness: three-valued logic, higher-valued logics and fuzzy set theory. In this paper, we look more closely at the first of these approaches, the use of three-valued logic, although other work currently under way is looking at the other approaches.

Table 1. Three-valued summary of aggregated nearness responses

\begin{tabular}{|c|c|c|c|c|c|c|c|c|c|c|c|c|c|c|c|c|c|c|c|c|c|c|c|}
\hline & Place & & & & & 5 & 6 & 7 & 78 & 9 & 10 & 11 & 12 & 13 & 14 & 15 & 16 & 17 & 18 & 19 & 20 & & 22 \\
\hline 1 & 24 Hour Reception & & & $?$ & $\perp$ & $?$ & $?$ & $\perp$ & $L \perp$ & $-\perp$ & $\perp$ & $?$ & $\perp$ & $?$ & $\perp$ & $\perp$ & $?$ & $?$ & $\perp$ & $?$ & $\perp$ & $T$ & $\perp$ \\
\hline 2 & Academic Affairs & ? & $\mathrm{T}$ & $?$ & ? & $\top$ & $?$ & ? & $\perp$ & - ? & $?$ & $\perp$ & $\perp$ & $\perp$ & $\perp$ & $\perp$ & ? & ? & $?$ & $\perp$ & $?$ & $?$ & $?$ \\
\hline 3 & Barnes Hall & ? & $\perp$ & $\mathrm{T}$ & $\perp$ & $?$ & $\perp$ & $\perp$ & $L \perp$ & $-\perp$ & $\perp$ & $\perp$ & $\perp$ & $\perp$ & $\perp$ & $\perp$ & $?$ & $\perp$ & $\perp$ & $?$ & $\perp$ & $\perp$ & $\perp$ \\
\hline 4 & Biological Sciences & ? & $?$ & $\perp$ & $\mathrm{T}$ & $?$ & $?$ & T & $\Gamma ?$ & ? ? & T & $\perp$ & $?$ & $?$ & $?$ & $\perp$ & $\perp$ & $?$ & $\mathrm{~T}$ & $\perp$ & $\top$ & $?$ & $?$ \\
\hline 5 & cellor's Building & ? & $\mathrm{T}$ & $?$ & $?$ & $\mathrm{~T}$ & $?$ & ? & $\perp$ & ? & $?$ & $\perp$ & $\perp$ & $\perp$ & $\perp$ & $\perp$ & $?$ & $?$ & $\perp$ & $\perp$ & $?$ & $?$ & $?$ \\
\hline 6 & Cha & ? & $\mathrm{T}$ & $?$ & $?$ & $?$ & T & - ? & ? & ? & $?$ & $\perp$ & $\perp$ & $?$ & $?$ & $\perp$ & $?$ & 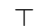 & $?$ & $\perp$ & $?$ & $\mathrm{~T}$ & $\perp$ \\
\hline 7 & Che & 1 & $?$ & & $\mathrm{~T}$ & $?$ & $?$ & T & $\Gamma ?$ & ? & $?$ & $\perp$ & $?$ & $\perp$ & $\perp$ & $\perp$ & $\perp$ & $?$ & $?$ & $\perp$ & $T$ & $\perp$ & $\bar{\top}$ \\
\hline 8 & Clo & & $\perp$ & $\perp$ & $?$ & $\perp$ & $\perp$ & -1 & $\mathrm{~L}$ & $-\perp$ & $?$ & $\perp$ & $?$ & $?$ & $\bar{T}$ & $?$ & $\perp$ & $?$ & $?$ & $\perp$ & $\perp$ & $?$ & $\perp$ \\
\hline 9 & Con & $\perp$ & $?$ & $?$ & $?$ & $?$ & $?$ & ? & $\perp$ & $-\mathrm{T}$ & $?$ & $\perp$ & $?$ & $\perp$ & $\perp$ & $\perp$ & $?$ & $\perp$ & $?$ & $\perp$ & $?$ & $\perp$ & T \\
\hline 10 & Ear & ? & $?$ & $\perp$ & $\top$ & $?$ & $?$ & ? & ? & ? & $\top$ & $\perp$ & $?$ & $?$ & $?$ & $\perp$ & $\perp$ & $?$ & $?$ & $\perp$ & $\top$ & ? & $?$ \\
\hline 11 & $\mathrm{H}$ & ? & $\perp$ & $\perp$ & $\perp$ & $\perp$ & $\perp$ & -1 & $L \perp$ & $-\perp$ & $\perp$ & $\bar{\top}$ & $\perp$ & $\mathrm{T}$ & $?$ & $?$ & $\perp$ & $\perp$ & $\perp$ & ? & $\perp$ & $?$ & $\perp$ \\
\hline 12 & The Oaks & $\perp$ & $\perp$ & $\perp$ & $?$ & $\perp$ & $\perp$ & ? & $\perp$ & $?$ & $\perp$ & $\perp$ & $\bar{T}$ & $\perp$ & $\perp$ & $\perp$ & $\perp$ & $\perp$ & $?$ & $\perp$ & $\perp$ & $\perp$ & $?$ \\
\hline 13 & Horwood Hall & $?$ & $\perp$ & $\perp$ & $\perp$ & $\perp$ & ? & $\perp$ & ? & $\perp$ & $\perp$ & T & $\perp$ & $\bar{\top}$ & $\bar{\top}$ & $\bar{\top}$ & $\perp$ & ? & $\perp$ & $?$ & $\perp$ & $\bar{\top}$ & $\dot{\perp}$ \\
\hline 14 & Keele I & ? & $\perp$ & $\perp$ & $\perp$ & $\perp$ & $?$ & $\perp$ & $\mathrm{L} T$ & $-\perp$ & $?$ & $?$ & $\perp$ & $?$ & $\top$ & $\top$ & $\perp$ & $?$ & $?$ & $\perp$ & $\perp$ & $?$ & $\perp$ \\
\hline 15 & Lakes & $\perp$ & $\perp$ & $\perp$ & $\perp$ & $\perp$ & $\perp$ & -1 & L? & $\perp$ & $\perp$ & $?$ & $\perp$ & $?$ & $\top$ & $\mathrm{T}$ & $\perp$ & $\perp$ & $?$ & $\perp$ & $\perp$ & $?$ & $\perp$ \\
\hline 16 & Leisure Centre & $\perp$ & $\perp$ & & $\perp$ & $?$ & $\perp$ & $-\perp$ & $L \perp$ & - ? & $\perp$ & $\perp$ & $?$ & $\perp$ & $\perp$ & $\perp$ & $\mathrm{T}$ & $\perp$ & $\perp$ & $\perp$ & $\perp$ & $\perp$ & $?$ \\
\hline 17 & Lib & $?$ & $?$ & $\perp$ & $?$ & $?$ & 丁 & $\Gamma ?$ & $?$ & ? & $\top$ & $?$ & $\perp$ & $?$ & $?$ & $?$ & $\perp$ & $\top$ & $?$ & $\perp$ & $?$ & $\top$ & $\perp$ \\
\hline 18 & Hall & $\perp$ & $\perp$ & $\perp$ & $?$ & $\perp$ & $\perp$ & L? & ? & ? & $?$ & $\perp$ & $\bar{\top}$ & $\perp$ & $?$ & $\perp$ & $\perp$ & $?$ & $T$ & $\perp$ & $?$ & $\perp$ & $?$ \\
\hline 19 & $\mathrm{Ob}$ & ? & $\perp$ & $?$ & $\perp$ & $\perp$ & $\perp$ & {$[\perp$} & $L \perp$ & $-\perp$ & $\perp$ & $?$ & $\perp$ & $?$ & $\perp$ & $\perp$ & $\perp$ & $\perp$ & $\perp$ & $\bar{\top}$ & $\perp$ & $?$ & $\perp$ \\
\hline 0 & $\mathrm{P}$ & $\perp$ & ? & $\perp$ & $\bar{T}$ & ? & ? & 7 & T? & $?$ & $\bar{\top}$ & $\perp$ & $?$ & $?$ & $\perp$ & $\perp$ & $?$ & $?$ & $?$ & $\perp$ & $\bar{\top}$ & $?$ & $?$ \\
\hline 2 & $\mathrm{~S}$ & $T$ & $?$ & & $?$ & $?$ & † & $\Gamma ?$ & ? & ? & $?$ & $?$ & $\perp$ & $\bar{T}$ & $?$ & $?$ & $\perp$ & $\top$ & $\perp$ & $?$ & $?$ & $\top$ & $\perp$ \\
\hline 22 & Visual Arts & & $\perp$ & $\perp$ & $?$ & $?$ & $\perp$ & L ? & $\perp$ & $-T$ & $?$ & $\perp$ & $?$ & $\perp$ & $\perp$ & $\perp$ & ? & $\perp$ & $?$ & $\perp$ & $?$ & $\perp$ & 1 \\
\hline
\end{tabular}

A $\chi^{2}$ (chi-squared) test was used to determine from the subjects' responses which places are regarded as near (denoted by $T$ ), not near (denoted by $\perp$ ), or undecided (denoted by ?), using a significance level of $\mathrm{P}=0.001$ (probability of $99.9 \%$ ). Table 1 summarises the aggregated responses of the 22 subjects, so for each column header $y$ we can read down the column all the places $x$ for which it is true, false or undecided to say $x$ is near to $y$. Taking $P$ as the set of places in our environment, the Campus, table 1 describes a three-valued nearness relation, 
$\nu$ on $P$, where for any two places $p, p^{\prime} \in P$ the nearness relations $p \nu p^{\prime}$ assumes one of the values $T, \perp$ or?

The notion of 'nearness' is a similarity (or tolerance) relation, where the formal properties of equivalence (reflexivity, symmetry and transitivity) are weakened. Nearness is assumed to be reflexive by definition and the leading diagonal in table 1 contains only $T$ values, although subjects were not actually asked questions of the form "is $x$ near to $x$ ". We can write a three valued reflexive property for the table, as in equation 1 below.

$$
\forall x \in P . x \nu x=\top
$$

The results exhibit the asymmetry that has already been noted as feature of human reasoning about nearness. From table 1 the Chapel is judged to be definitely near to Academic Affairs, but Academic Affairs is not definitely near to the Chapel. While the Clock House is definitely not near to Physics, the converse is not the case. Sadalla et al. [21] also observed this asymmetry, and attributed it to the relative prominence or importance of different features, although [25] provides evidence that scale effects may have a significant bearing upon the asymmetry. However, the results do preserve a degree of symmetry. There are no places in table 1 where $x$ is judged to be near to $y$ and $y$ is judged to be not near to $x$. Consequently, we can write down a weak symmetry property that does hold for all places, given in equation 2 below.

$$
\forall x, y \in P . x \nu y=\top \text { implies } y \nu x \neq \perp
$$

While as might be expected the relation is not transitive, it does also exhibit a degree of transitivity. The relatively high significance level used was chosen as the lowest tested value of $\mathrm{P}$ for which the results preserved weak transitivity, defined in equation 3 below. In short, the study found no places at the 0.001 significance level, for which $x$ was near to $y$ and $y$ was near to $z$ but $x$ was not near to $z$, so the relation $\nu$ satisfies the property in equation 3

$$
\forall x, y, z \in P .(x \nu y=\top \text { and } y \nu z=\top) \text { implies } x \nu z \neq \perp
$$

In summary, despite being derived from subjective data about which places are understood to be near to each other in Keele University campus, the data in its three valued form does exhibit some formal structure in terms of its reflexivity, weak symmetry and weak transitivity. The following section looks at how further cognitively plausible formal structure can be found in the data using data mining techniques.

\section{Data mining analysis}

In pursuit of greater integration between the computational and cognitive approaches to reasoning about nearness, this section looks at the use of data mining techniques in combination with the experimental data. The following two 
sections explore two related computational structures derived using the experimental data: faithful minimal frameworks and decision trees. These two computational structures can in turn be related to two analogous cognitive structures: landmarks and hierarchies.

\subsection{Landmarks and faithful minimal frameworks}

Landmarks can be defined as "... places whose locations are relatively better known and that serve to define the location of adjacent points" ([21] p. 517). Exactly which features in an environment constitute landmarks for a particular individual may depend upon a range of social, cultural, physical and spatial factors not covered in this experiment. Fisher and Orf [5] do attempt to relate subjective ideas about nearness to certain social characteristics of the experimental subjects, but in this study such effects are not considered. By looking at which places "serve to define the location of adjacent points" it should be possible to derive some candidate sets of landmarks. The existence of landmarks should be expressed as dependencies within the data, where non-landmark places can be discriminated simply with reference to their locations relative to the landmarks.

Happily, the task of finding such dependencies can be achieved relatively simply using data mining techniques for finding and eliminating dependencies in data. Let $P$ be the set of 22 reference places. We use the term framework to refer specifically to sets of reference places, $A \subseteq P$. In table 1 , any two places that have exactly the same nearness values with respect to the framework $P$ can be considered indiscernible from each other. For the full table of data, every place can be uniquely identified and no two places have exactly the same relationship to the framework $P$ (no two rows are identical). One question is whether there are any smaller frameworks $A \subset P$ for which it is still possible to uniquely identify every place in the data set. Formally we can define an equivalence relation $\tilde{A}$ on a subset of places $U \subseteq P$ for any framework $A \subseteq P$, as in equation 4 below.

$$
\tilde{A}=\left\{\left(x, x^{\prime}\right) \mid x, x^{\prime} \in U \text { and } \forall y \in A . x \nu y=x^{\prime} \nu y\right\}
$$

A nonempty set $A \subseteq P$ is termed a dependent set if for some proper subset $A^{\prime} \subset A$, both $A$ and $A^{\prime}$ have the same equivalence relation $\left(\tilde{A}=\tilde{A}^{\prime}\right)$, otherwise $A$ is termed a minimal set ([16] p. 163). Here the terms dependent and minimal frameworks are used in place of dependent and minimal sets to highlight the fact that the discussion concerns sets of reference places. A desirable property of minimal frameworks is that they should still allow us to distinguish between every place in the data. This property is termed faithfulness and occurs when $\forall\left(x, x^{\prime}\right) \in \tilde{A} . x=x^{\prime}$. Faithful minimal frameworks provide us with the smallest sets of reference places with respect to which it is still possible to distinguish every place in the study.

Finding faithful minimal frameworks using an exhaustive search is computationally intensive, but for this relatively small data set entirely possible. For a set of 22 reference places there are just over 4 million possible subset combinations to check. An exhaustive search of the data, achieved using Java code 
written specially for the task, revealed 1951 faithful minimal frameworks. The three smallest faithful minimal frameworks contained only 5 reference places. To illustrate, knowledge of location (in terms of near, not near or undecided) with respect to just five reference places (for example, the framework 24 Hour Reception, Barnes Hall, Library, Lindsay Hall, Visual Arts $\}$ ) is enough to uniquely identify each of the 22 places. While faithful minimal frameworks are clearly not the same as cognitive reference points or landmarks, they go some way towards closer integration of cognitive and computational approaches to nearness. Purely on the basis of the structure of the data, irrespective of social or physical influences, the faithful minimal frameworks represent good candidate sets of landmarks since they are derived from cognitive experimental data and can "serve to define the location of adjacent points".

\subsection{Information content}

Unfortunately, the exhaustive search for faithful minimal frameworks is computational intractable, requiring $O\left(2^{n}\right)$ time to complete. Since the time complexity of the algorithm increases exponentially with the number of locations used, an exhaustive search for faithful minimal frameworks would not be practical for analysis large data sets. However, information theory is commonly applied as a heuristic that can significantly reduce the solution space in data mining, for example in the well known ID3 algorithm (see [20]). Knowledge about nearness to a single reference place will never allow all the places in the study to be distinguished. However, it does allow us to distinguish between some places. Shannon's information content [22] offers a mechanism for quantifying how much an individual reference place allows us to distinguish between the different places in the study. Information content can provide a measure of how much information is gained by a knowing about nearness to a particular reference place. It is possible to quantify the information content $I(p)$ for particular reference place $p \in P$ a with respect to a set of places $U \subseteq P$ using equation 5, after [22], where $|X|$ denotes the cardinality of the set $X$.

$$
I(r)=\sum^{n \in\{T, ?, \perp\}}-\frac{|\{x \in U \mid x \nu r=n\}|}{|U|} \log _{2} \frac{|\{x \in U \mid x \nu r=n\}|}{|U|}
$$

Information content is additive, so for a framework $A \subseteq P$ we can calculate the total information content $I(A)$ by simply taking the sum of information content for each element of the set, as in equation 6 .

$$
I(A)=\sum^{a \in A} I(a)
$$

By selecting those reference places that maximise the information gained about the data set, it should be possible to obtain a reasonably small faithful framework at the same time as searching only a small portion of the data. Again, Java code was written to accomplish this task. There are a range of slightly different ways of using information content (see [16]) and depending on the 
specific details of the heuristic used, the algorithm applied to the data in table 1 resulted in a faithful dependent (not minimal) framework containing about 10 reference places. If these 10 locations are used to constrain a further exhaustive search for faithful minimal sets, 4 of the 10 locations can be eliminated. Although this is a still a sub-optimal result, resulting in a faithful minimal framework with cardinality 6 , the process is much faster than a full exhaustive search. The time complexity of data mining algorithm is $O(n)$ in the worst case, and may often be much better. In practice, when applied to the data for the 22 locations used in this study, the algorithm required less than a second or two to run on a standard PC, compared with more than 3 hours needed to complete an optimised exhaustive search.

\subsection{Decision trees and hierarchies}

A further use of information content is in the production of decision trees to describe the data set. Decision trees are an intuitive hierarchical structure for representing the data in table 1 in a much more compact, easily accessible form. While decision trees do not possess the same semantics as the hierarchies observed in $[23,12]$, they are analogous to such hierarchies and consequently represent a potentially useful, cognitively plausible computational structure. By recursively comparing the information content of each attribute in a faithful minimal framework, it is possible to build a decision tree that represents the framework with the most important information rich reference places nearer the root of the tree, and the least important information sparse reference places nearer the leaves. For some set of places $U \subseteq P$ and framework $A \subseteq P$, we can generate a decision tree using the following algorithm, after [17].

1. If the set of places $U$ has only one element or if $A=\varnothing$, create new leaves in the decision tree with the values $u \in U$.

2. Otherwise for each reference place $a \in A$ calculate the information gain associated with that place using the equation $I(A)-I(A \backslash\{a\})$. Select the reference place $r$ associated with the largest information gain.

3. Using the selected reference place $a \in A$ partition $U$ into disjoint sets $U_{n}=$ $\{x \in U \mid x \nu a=n\}^{n \in\{T, ?, \perp\}}$. A new decision node is then created to represent $a$ and the algorithm is reiterated for each $U_{n} \neq \varnothing$ using $A \backslash\{a\}$ as the framework.

The decision trees produced by this algorithm provide a more compact, intuitive representation of the qualitative nearness information than data tables such as in table 1 . An example decision tree for the faithful minimal framework mentioned in $\S 4.1$ (1, 24 Hour Reception; 3, Barnes Hall; 17, Library; 18, Lindsay Hall; 22, Visual Arts) is given in figure 1. The decision nodes (white boxes) correspond to decisions about whether a place is near one of the five reference places in the framework. The leaf nodes (black boxes) correspond to the 22 places which can be distinguished. Leaf node 21 (Student Union), for

example, can be uniquely identified as the only place that is not near 22 (Visual 


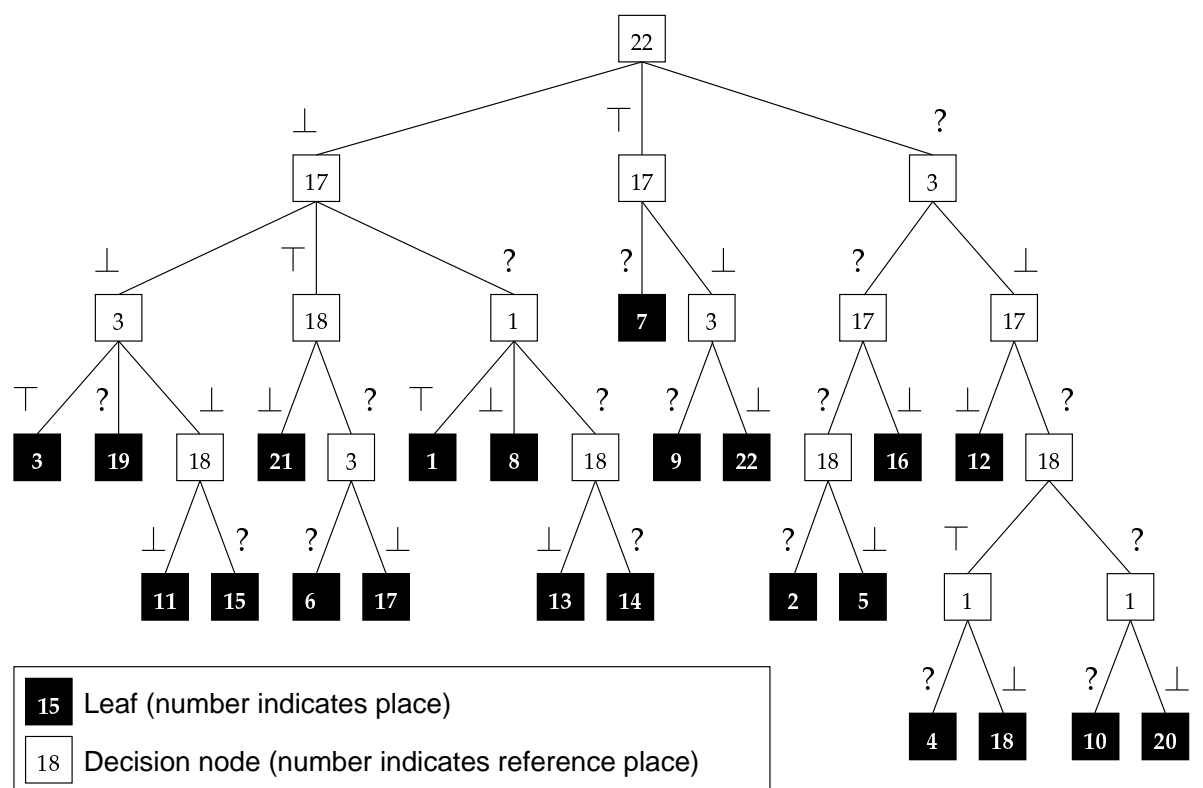

Fig. 1. Example nearness decision tree (numbers correspond to table 1)

Arts), near 17 (Library) and not near 18 (Lindsay Hall). Decision nodes nearer the root of the tree yield greater information gain than those nearer the leaves. It is worth noting that while 5 places are needed to distinguish every place on Campus, most places (18) can be uniquely identified with reference to between just 2 and 4 places.

\section{Qualitative vector spaces}

The previous section indicates that data mining techniques can go some way towards integrating computational and cognitive aspects of a three-valued nearness relation. In this section we will construct a qualitative vector space (QVS), using as a framework for the space a subset of places in the environmental space (the Campus, in our example). Places in the environmental space will then be capable of representation as qualitative vectors with respect to the framework. The QVS can itself be given a nearness structure. One of the key issues is how well the QVS with its nearness relation represents the environmental space and its nearness relation. We now set up the formal apparatus.

\subsection{Qualitative coordinates}

Let $\mathcal{E}$ be a given environmental space, and $P$ be the set of reference places in the space. The experimental data has provided a three-valued nearness relation 
$\nu$ on $P$. Let $A \subseteq P$ be the framework for the coordinate system. For each place $p \in P$, we can define the qualitative coordinates of $p$ with respect to $A$, denoted $\vec{p}_{A}$, as follows:

$$
\vec{p}_{A}=\{(a, p \nu a) \mid a \in A\}
$$

We refer to $\vec{p}_{A}$ as a qualitative vector with respect to $A$. If the framework $A$ is understood, then the qualitative coordinates of $p$ are just written $\vec{p}$.

It will also be useful to have a notation for a collection of qualitative vectors. Let $Q \subset P$. Then, define the qualitative vector set $\vec{Q}_{A}$ as follows:

$$
\vec{Q}_{A}=\left\{\vec{q}_{A} \mid q \in Q\right\}
$$

Again, if the framework $A$ is understood, then we can drop the subscript.

\subsection{Qualitative vector space}

Given a set of places $P$, a three-valued nearness relation $\nu$ defined on $P$, and a framework $A \subseteq P$, we have defined a qualitative vectors set $\vec{P}_{A}$. Assume for the remainder of this section that $A$ is fixed. We can give the qualitative vector set $\vec{P}$ a structure by defining on it a nearness relation $\vec{\nu}$, as follows. Let $\vec{x}, \vec{y} \in \vec{Q}$, then

$\vec{x} \vec{\nu} \vec{y}$ if and only if $\nexists a \in A .((x \nu a=\top \wedge y \nu a=\perp) \vee(x \nu a=\perp \wedge y \nu a=\top))(9)$

The pair $\overrightarrow{\mathcal{P}}=<\vec{P}, \vec{\nu}>$ is termed a qualitative vector space (QVS). The QVS $\overrightarrow{\mathcal{P}}$ has some general properties, detailed below.

Reflexive property: $\forall \vec{x} \in \vec{P} . \vec{x} \vec{\nu} \vec{x}$

Symmetric property: $\forall \vec{x}, \vec{y} \in \vec{P} . \vec{x} \vec{\nu} \vec{y}=\vec{y} \vec{\nu} \vec{x}$

The symmetric property of $\vec{\nu}$ shows up an immediate difference between $\vec{\nu}$ and $\nu$, as $\nu$ may well not be symmetric (see $\S 3$ ). The next subsections define properties that relate the closeness of fit between the environmental space and its representation as a QVS. The properties are all expressed in terms of the QVS, but we can equally talk of the properties relating to the frames underlying the QVS.

Faithfulness A QVS provides a faithful representation of a nearness space if the representation provides an injective function from the vector set to the coordinate set. The intuition behind faithfulness is that the representation will represent different places with different sets of coordinates, as so different places can be distinguished in the vector space. Thus, the property of faithfulness for QVS corresponds to the property of faithfulness in experimental data, introduced in $\S 4$. In detail, we have the following definition.

Definition: The QVS $\overrightarrow{\mathcal{P}}$ is faithful if and only if the following condition holds:

$$
\forall x, y \in P . \vec{x}=\vec{y} \Rightarrow x=y
$$


Adequacy The next properties of a QVS concern its ability to predict nearness and non-nearness relations in the environmental space. A QVS is adequate ${ }^{+}$if whenever two vectors are near, then the places that they represent are near. In a similar way, a QVS is adequate ${ }^{-}$if whenever two vectors are not near, then the places that they represent are not near. We need to remember that the relation $\vec{\nu}$ is Boolean while $\nu$ takes values from 3 -valued logic. Formally, we have the following definitions.

Definition: The QVS $\overrightarrow{\mathcal{P}}$ is adequate ${ }^{+}$if and only if the following condition holds:

$$
\forall x, y \in P . \vec{x} \vec{\nu} \vec{y} \Rightarrow x \nu y=\top
$$

Definition: The QVS $\overrightarrow{\mathcal{P}}$ is adequate ${ }^{-}$if and only if the following condition holds:

$$
\forall x, y \in P . \neg \vec{x} \vec{\nu} \vec{y} \Rightarrow x \nu y=\perp
$$

We can easily prove that if there are any occurrences of uncertainty in the relation $\nu$, then no QVS can be both adequate ${ }^{+}$and adequate $^{-}$. To see this, suppose that QVS $\overrightarrow{\mathcal{P}}$ is adequate ${ }^{+}$and adequate $^{-}$. Suppose also that $\exists x, y \in P$ such that $x \nu y=$ ?. Then, Equation 11 implies that $\neg \vec{x} \vec{\nu} \vec{y}$, while Equation 12 implies that $\vec{x} \vec{\nu} \vec{y}$. As $\vec{\nu}$ is a Boolean relation, we have a contradiction.

We can weaken the adequacy definitions to make them more in accord with our nearness data.

Definition: The QVS $\overrightarrow{\mathcal{P}}$ is weakly adequate ${ }^{+}$if and only if the following condition holds:

$$
\forall x, y \in P . \vec{x} \vec{\nu} \vec{y} \Rightarrow x \nu y \neq \perp
$$

Definition: The QVS $\overrightarrow{\mathcal{P}}$ is weakly adequate ${ }^{-}$if and only if the following condition holds:

$$
\forall x, y \in P . \neg \vec{x} \vec{\nu} \vec{y} \Rightarrow x \nu y \neq \top
$$

Definition: The QVS $\overrightarrow{\mathcal{P}}$ is weakly adequate if and only if it is both weakly adequate $^{+}$and weakly adequate ${ }^{-}$.

Note: It is useful to think of faithfulness and adequacy properties applying to the underlying framework of the faithful or adequate QVS. This leads to the following concepts of minimal and maximal frameworks.

Minimal and maximal frameworks Clearly, if a framework $A$ is faithful, and if $A \subseteq A^{\prime}$, then framework $A^{\prime}$ is also faithful. Therefore, minimal faithful sets can be defined as follows.

Definition: The framework $A$ is minimal faithful if 
1. $A$ is faithful.

2. $A^{\prime} \subset A$ implies that $A^{\prime}$ is not faithful.

It is also easy to see that if framework $A$ is (weakly) adequate ${ }^{+}$, and if $A \subseteq A^{\prime}$, then framework $A^{\prime}$ is also (weakly) adequate ${ }^{+}$. Similarly, if framework $A$ is (weakly) adequate ${ }^{-}$, and if $A \supseteq A^{\prime}$, then framework $A^{\prime}$ is also (weakly) adequate $^{+}$. It therefore makes sense to define minimal (weakly) adequate ${ }^{-}$QVS and maximal (weakly) adequate ${ }^{+}$QVS in the obvious way.

Definition: The framework $A$ is minimal (weakly) adequate $e^{+}$if

1. $A$ is (weakly) adequate ${ }^{+}$.

2. $A^{\prime} \subset A$ implies that $A^{\prime}$ is not (weakly) adequate ${ }^{+}$.

Definition: The framework $A$ is maximal (weakly) adequate ${ }^{-}$if

1. $A$ is (weakly) adequate ${ }^{-}$.

2. $A^{\prime} \supset A$ implies that $A^{\prime}$ is not (weakly) adequate ${ }^{-}$.

\subsection{Experimental qualitative vector spaces}

Having defined the nature and properties of qualitative vector spaces above it is worth reviewing what sort of qualitative vector space the experimental data describes. The definition of faithfulness in equation 10 corresponds to the definition given in $\S 4.1$, and the data mining techniques used in $\S 4.1$ uncovered 1951 such faithful (minimal) frameworks within the data. More than half the combinations of sets exhibits adequacy in at least one of the forms defined in equations 11-14, and more than 25000 of these frameworks are at the same time weakly adequate ${ }^{+}$ and weakly adequate ${ }^{-}$. However, there are no sets which are adequate ${ }^{+}$. While it is possible for such sets to exist, they are heavily constrained. A framework $A \subseteq P$ can only be adequate ${ }^{+}$if for all places $x, y \in P$ where $\vec{x}_{A} \vec{\nu} \vec{y}_{A}$ then $x \nu y=y \nu x=\top$ also hold. The tendency against strong symmetry in the environmental space (see §3) militates against such frameworks occurring. There are just three non-trivial frameworks that are adequate ${ }^{-}$, one of which is maximal (\{Barnes hall, Observatory\}). Significantly, Barnes Hall and the Observatory are peripheral locations not considered to be near to anywhere else, nor is anywhere considered to be near them (except reflexively themselves). Consequently, the framework $A=\{$ Barnes Hall, Observatory $\}$ possesses the rare symmetrical property that for all locations $x, y \in P$ if $\vec{x}_{A} \vec{\nu}_{A}$ then $x \nu y=y \nu x=\perp$ also hold.

Finally, a search for the smallest frameworks which are weakly adequate ${ }^{+}$, weakly adequate ${ }^{-}$and faithful yielded just 47 dependent frameworks with a range of cardinalities from 10 to 16 places (eg \{Academic Affairs, Health Centre, Holly Cross/The Oaks, Keele Hall, Leisure Centre, Library, Lindsay Hall, Physics, Student Union, Visual Arts\}). The size of these frameworks suggests that the compromise needed to retain all three properties, weak adequacy ${ }^{+}$, weak adequacy - and faithfulness leads to a degree of redundancy in the frameworks, 
making them relatively large when compared with the minimal faithful frameworks. However, it is still useful to note that there do exist frameworks within the experimental data the exhibit these three properties together. Computationally, finding such sets is relatively efficient. An exhaustive search is easily optimised using the properties of minimal (weak) adequacy ${ }^{+}$and minimal faithfulness given above. From the minimally weakly adequate ${ }^{+}$property it follows that for some $A^{\prime} \subset A$ where $A$ is known not to be weakly adequate ${ }^{+}$then $A^{\prime}$ is not weakly adequate $^{+}$. A similar optimisation is suggested by minimal faithfulness. While an unoptimised exhaustive search of the data, written using Java, required more than 24 hours to complete, constraining the search using the above optimisations reduced the search time to just over 30 minutes. A further possible optimisation might be based on the minimally weakly adequate ${ }^{-}$sets, where $A^{\prime} \supset A$ and $A$ is known not to be weakly adequate ${ }^{-}$then $A^{\prime}$ is not weakly adequate. However, in practice the additional complexity introduced into the search by the additional constraint tended to increase rather than decrease the overall search time.

\section{Discussion}

The analyses presented above indicate how cognitive and computational approaches to qualitative spatial relations like nearness can be more closely integrated. The treatment in this paper provides an approach that is formally well founded and also cognitively plausible, in the sense of its derivation from human subject experimentation. The main contribution of the paper has been to develop the idea of frameworks, which provide as full as possible information about nearness relations within an environmental space. The frameworks form the basis of a QVS, that may exhibit two important formal properties: faithfulness and adequacy. These properties relate directly to important features of human perception of an environmental space. Faithfulness concerns the discrimination of different places, such that if two places are perceived as distinct, then their nearness relations to places in the framework are also distinct. Adequacy relates to the ability to extrapolate knowledge about nearness relations in a framework to the perceived nearness relations for the complete place set.

The experimental data was found to exhibit several frameworks that are faithful, weakly adequate ${ }^{+}$and weakly adequate ${ }^{-}$. The property of weak adequacy ${ }^{+}$ ensures that places that are near with respect to the framework are at least near or undecided in the perceived space. Conversely, the property of weak adequacy ${ }^{-}$ ensures that places that are not near to each other with respect to the framework are not perceived as near to each other. In the light of $\S 5$, where it is shown that it is formally impossible for a framework to have all the strongest properties at the same time, this result indicates that the experimental data does retain interesting formal properties.

While the results do indicate a cognitively plausible formal model of nearness, it is not yet clear to what extent the results are repeatable in different contexts. Consequently, future experimental work will study how the properties of QVS 
vary under different conditions. Planned further work includes studies of the effects of using:

- different human subjects, such as random surveys of people using Keele University campus.

- different environmental spaces, such as environmental spaces at different scales (eg regional, national or international scales) and dimensions (eg road or rail networks).

- different qualitative spatial relations, for example qualitative direction, which in turn might serve as a basis for an integrated formal model of qualitative space.

In addition to further experimentation with human subjects, future work will be in three directions. First, more formal work both on the underlying theory of qualitative vector spaces is planned and on different representations of the experimental data (for example using higher valued logics or fuzzy set theory). Second, further computational development will be pursued, including refinement of the data mining algorithms for finding suitable reference sets. Finally, finding suitable framework sets is highly desirable in many practical applications, from wayfinding to location specification. Work currently in progress, based on this theoretical work, aims to address the development of a prototype software able to assist in such a wayfinding and location specification scenario.

\section{Acknowledgements}

The authors are grateful to Peter Smith for helpful discussions on appropriate statistical methods and to Tony Cohn for useful suggestions concerning the construction of decision trees. This research is supported by the UK EPSRC under grant GR/M 56685 "Managing vagueness, uncertainty and granularity in spatial information".

\section{References}

1. A.G. Cohn, A hierarchical representation of qualitative shape based on connection and convexity, Spatial Information Theory: A Theoretical Basis for GIS (A.U. Frank and W. Kuhn, eds.), Lecture Notes in Computer Science, no. 988, SpringerVerlag, Berlin, 1995, pp. 311-326.

2. A.G. Cohn and N.M. Gotts, The 'egg-yolk' representation of regions with indeterminate boundaries, Geographic Objects with Indeterminate Boundaries (Burrough, P.A. and Frank, A.U., eds.), GIS Data 2, Taylor and Francis, London, 1996.

3. M.J. Egenhofer and R.D. Franzosa, Point-set topological spatial relations, International Journal of Geographical Information Systems 5 (1991), no. 2, 161-174.

4. P.F. Fisher, Sorites paradox and vague geographies, Fuzzy Sets and Systems 113 (2000), no. 1, 7-18.

5. P.F. Fisher and T.M. Orf, An investigation of the meaning of near and close on a university campus, Computers, Environment and Urban Systems 15 (1991), 23-35. 
6. A.U. Frank, Qualitative spatial reasoning about distances and directions in geographic space, Journal of Visual Languages and Computing 3 (1992), 343-371.

7. M. Gahegan, Proximity operators for qualitative spatial reasoning, Spatial Information Theory: A Theoretical Basis for GIS (A.U. Frank and W. Kuhn, eds.), Lecture Notes in Computer Science, no. 988, Springer-Verlag, Berlin, 1995, pp. 31-44.

8. _ Experiments using context and significance to enhance the reporting capabilities of GIS, Spatial Information Theory: A Theoretical Basis for GIS (S.C. Hirtle and A.U. Frank, eds.), Lecture Notes in Computer Science, no. 988, SpringerVerlag, Berlin, 1997, pp. 485-496.

9. D. Hernández, E. Clementini, and P. Di Felice, Qualitative distances, Spatial Information Theory: A Theoretical Basis for GIS (A.U. Frank and W. Kuhn, eds.), Lecture Notes in Computer Science, no. 988, Springer-Verlag, Berlin, 1995, pp. 4557.

10. S.C. Hirtle, Representational structures for cognitive spaces: trees, ordered trees and semi-lattices, Spatial Information Theory: A Theoretical Basis for GIS (A.U. Frank and W. Kuhn, eds.), Lecture Notes in Computer Science, no. 988, Springer-Verlag, Berlin, 1995, pp. 327-340.

11. S.C. Hirtle and T. Gärling, Heuristic rules for sequential spatial decisions, Geoforum 23 (1992), no. 2, 227-238.

12. S.C. Hirtle and J. Jonides, Evidence of hierarchies in cognitive maps, Memory and Cognition 13 (1985), no. 3, 208-217.

13. S.C. Hirtle and M.F. Mascolo, Effect of semantic clustering on the memory of spatial locations, Journal of Experimental Psychology: Learning, Memory and Cognition 12 (1986), no. 2, 182-189.

14. G.F. Ligozat, Qualitative triangulation for spatial reasoning, Spatial Information Theory: A Theoretical Basis for GIS (A.U. Frank and I. Campari, eds.), Lecture Notes in Computer Science, no. 716, Springer-Verlag, Berlin, 1993, pp. 54-68.

15. D. Medyckyj-Scott and M. Blades, Human spatial cognition: its relevance to the design and use of spatial information systems, Geoforum 23 (1992), no. 2, 215-226.

16. T. Munakata, Fundamentals of the New Artificial Intelligence, Springer-Verlag, 1998.

17. J.R. Quinlan, Learning efficient classification procedures and their application to chess end games, Machine Learning: An Artificial Intelligence Approach (R.S. Michalski, J.G. Carbonell, and T.M. Mitchell, eds.), Morgan Kauffmann, California, 1983, pp. 463-482.

18. V.B. Robinson, Interactive machine acquisition of a fuzzy spatial relation, Computers and Geosciences 16 (1990), no. 6, 857-872.

19. __ Individual and multipersonal fuzzy spatial relations acquired using humanmachine interaction, Fuzzy Sets and Systems (2000), no. 113, 133-145.

20. S. Russell and P. Norvig, Artificial Intelligence: A Modern Approach, Prentice Hall, New Jersey, 1995.

21. E.K. Sadalla, W.J. Burroughs, and L.J. Staplin, Reference points in spatial cognition, Journal of Experimental Psychology: Human Learning and Memory 6 (1980), no. 5, 516-528.

22. C.E. Shannon, A mathematical theory of communication, The Bell System Technical Journal 27 (1948), 379-423, 623-656.

23. A. Stevens and P. Coupe, Distortions in judged spatial relations, Cognitive Psychology 10 (1978), no. 4, 422-437.

24. B. Tversky, Distortions in cognitive maps, Geoforum 23 (1992), no. 2, 131-138.

25. M.F. Worboys, Nearness relations in environmental space, International Journal of Geographical Information Science (2001), In press. 


\section{University Library}

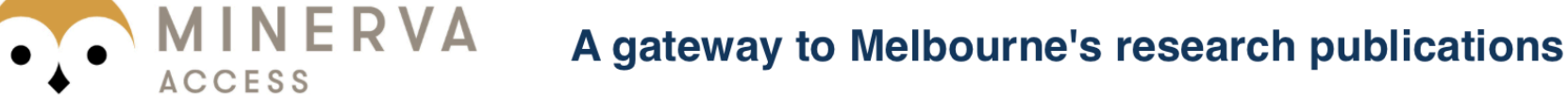

Minerva Access is the Institutional Repository of The University of Melbourne

Author/s:

DUCKHAM, MATT;WORBOYS, MICHAEL

Title:

Computational structure in three-valued nearness relations

Date:

2001

Citation:

Duckham, M., \& Worboys, M. (2001). Computational structure in three-valued nearness relations. In, D. Montello (Eds.) Lecture Notes in Computer Science 2205 (pp. 76-91). Springer.

Publication Status:

Published

Persistent Link:

http://hdl.handle.net/11343/33602 